\title{
GREEN SCORE: DEVELOPING A MEASUREMENT MODEL FOR SUSTAINABLE PEDESTRIAN-FRIENDLY ENVIRONMENT BASED ON SPACE SYNTAX
}

\author{
Yongjin Joo $^{1}$, Young Il KIM ${ }^{2}$, and Tae-Ho Kim${ }^{3 *}$ \\ ${ }^{1}$ Institute of Urban Sciences, University of Seoul, Seoul, Korea \\ ${ }^{2}$ Graduate School of Urban Studies, Hanyang University, Seoul, Korea \\ ${ }^{3}$ Institute of Urban Sciences, University of Seoul, Seoul, Korea \\ *Corresponding author (traffix@uos.ac.kr)
}

\begin{abstract}
Pedestrian-oriented transportation has recently emerged as an important issue to supplement transportation modes as a method to reduce traffic accidents and environmental pollution. Compared to the increasing attention, practical studies considering the transportation environment in Korea are very limited. As a first step for studying the service level of pedestrian environment, it is necessary to develop an objective and systematic evaluation scheme. This paper aims to develop the 'Green Score', an evaluation system, which can be used to compute and evaluate the service level of pedestrian environment. In this study, we focused on pedestrian axes associated with vehicle crosses for the evaluation. We applied the depth-based index used in space syntax theory in assessing the space structure in terms of the connectivity. For setting the weight values of evaluation criteria obtained from survey, we employed AHP (Analytic Hierarchy Process). The computed scores using these criteria are classified and normalized according to the levels of pedestrian dependency and, finally, displayed on a map. The proposed Green Score system is expected to be applicable in the visualization of pedestrian movements and the evaluation of convenience levels of pedestrian environment.
\end{abstract}

Keywords: Walking Environmental Score, Space Syntax, AHP(Analytic Hierarchy Process), Web GIS Service

\section{INTRODUCTION}

For the field of city planning and transportation, sustainable development that considers the two concepts of development and conservation in harmony has recently been gaining popularity. The key to urban planning based on this new planning paradigm is constructing high-density cities tied to complex land use patterns and public transportation systems. As a method of dealing with problems caused by other means of transportation, such as traffic accidents or pollution, there is a growing use of green methods of transportation. An urban settlement environment that restrains an auto-based life style and encourages walking activities must be considered in urban planning and design. In particular, though research on Transit-Oriented Development (TOD) has recently emerged as an issue, most domestic studies have not been addressed comprehensive analysis or concrete inducement technique when it comes to effects of TOD. Therefore, decision support system which is able to create assistance in policy system by analyzing quantitative effect concerning major plan factors of TOD such as transit mode, LOS, density, diversity of land use, and pedestrian-friendly urban design focusing a transit center.

There have been a number of studies on pedestrian-friendly activities and their support of urban environments, disclosing their measurement indexes $[5,6,7,8,9,11,12$, 13]. Discussions of the concept of the pedestrian environment have focused on how many physical attractions a place has to induce walking. In other words, the major measurement parameters to explain pedestrianfriendly environments in street design are accessibility, connectivity, continuity, convenience, and safety. Among analytical methodologies, surveys on pedestrians are widely used to evaluate the pedestrian movement space. 
The current qualitative methodology, however, may have the downside of distorted responses related to satisfaction and uneven measurement results resulting from respondents' different experiences. To resolve the problems in current methodologies and qualitative evaluation indexes, quantifying the pedestrian environment index using a GIS database and space analysis, as well as quantitatively evaluating the street environment for walking, is critical in the construction of a pedestrianfriendly environment.

Therefore, the objective of this study is to develop a walkability evaluation scheme called Green Score that simplify pedestrian environment elements depending on types of walking space with a quantitative index, and evaluating and visualizing the overall degree (in terms of pleasantness and aesthetics) of pedestrian friendliness of the environment, as well as pedestrians' convenience and accessibility. We explain how we designed the depth-based index used in space syntax theory in assessing the space structure in terms of the connectivity, taking into consideration in existing methods related with pedestrian level of services in the following sections. As a case study, the walk score has been calculated to evaluate the spatial characteristics of pedestrian environments along with the facility characteristics of pedestrian networks in relation to the major subway stations, Sadadng, in Seoul, Korea. Finally, we draw some findings in the conclusion

\section{Design of Walking Environment Indices}

This study has measured distribution of the traffic facilities and amenities as well as connectivity of the pedestrian networks quantitatively in order to perform comprehensive evaluation of the pedestrian environments. To design a pedestrian environment index, quantifiable parameters such as mobility, safety, convenience, pleasantness, and environment-friendliness were organized. Following parameters were selected and they are viewed to have the potential to be used as indexes for the development of GIS.

\section{1) Walk Accessibility}

In order to measure the connectivity of pedestrian networks, we have developed qualitative convenience factors using
GIS and quantitative network connectivity indices in the Space Syntax Technique. Specifically, we used intelligibility, which is able to consider integration and connectivity from the outputs of the model simultaneously to quantify the levels of recognition and connectivity of the pedestrian networks.

The Space Syntax is based on spatial cognition and spatial behavior [3, 10]. Hart and Moor [2] defined spatial cognition as the cognitive representation concerned with spatial structure, factor and relative relation. Burnett [1] defined perception and cognition of the relation between the human and the surrounding environment as an intervening psychological process of human behavior. The main factor of spatial cognition and cognitive representation is sight. The basic factor of Space Syntax comes from visibility, how far we can perceive, and accessibility.

As shown in Figure 1, this study follows the procedure outlined by Hillier and Hanson [4]. First, make a convex map (b), which is composed of convex spaces, from the original settlement. Convex space is defined as a space with no line drawn between any two points in the space goes outside the space. An axial map can be made from the convex map. The fewest number of long axial lines have been drawn along the convex spaces which represent all public routes through which people can see and move. The longest possible lines of sight have been used to define the axes (d).
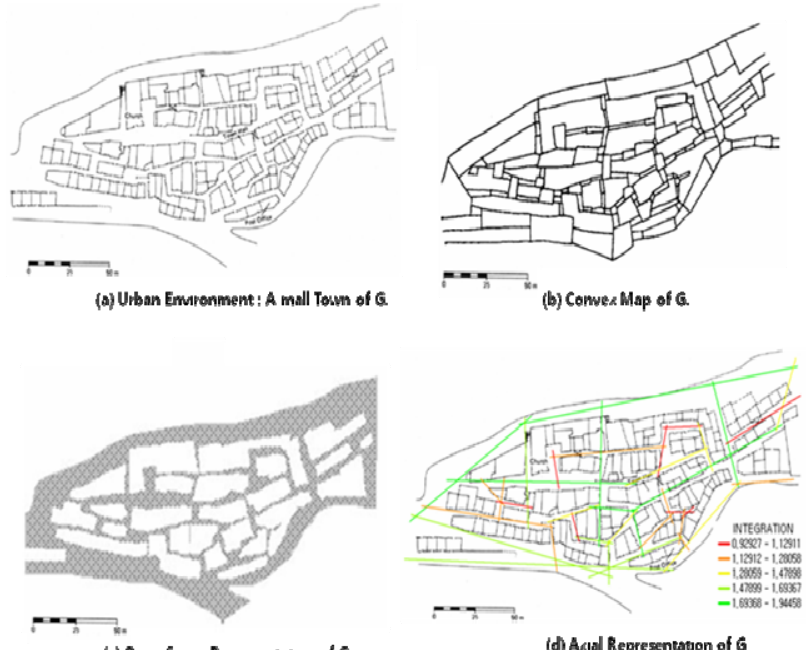

(c) Open Space Representaton of 6

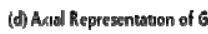

Fig.1 The construction of the axial map 
Movement can be described in an abstracted form using its topology. Topological description helps focus on the structural relationship among units. In other words, this model is a space analysis technique to handle with movement from connectivity relation of the spaces from probability aspect, which is able to measure the possibility of space occupation by the user and predict the traffic for the possibility of space occupation.

Integration refers to the one calculated by summating space depths of individual routes by assuming the entire axial lines expressed on the axial line chart within the objective range of analysis with their start and end points and assuming that it is possible to move from one axial line to the other axial line. As the integration has high value, movement steps from any space to the space become less and allow easy access. Connectivity is a variable a level of connectivity of spaces, representing the number of axial lines to be directly connected. Intelligibility means the extent to recognize the partial space from the entire space or the entire space from the partial space. Intelligibility of a space is expressed as correlation of Integration and Connectivity. Therefore, Pedestrian movement can be described using network of simple lines without considering the details such as sizes of forms, number of people and speed of movement. What's more, the walk accessibility parameter evaluates the connectivity and accessibility of street segments' structural space characteristics.

\section{2) Intersection Safety}

Intersection safety parameters include the perception of a vehicle coming toward a pedestrian at a crosswalk, and crosswalk function is measured for mobility and accessibility. Ladder Crosswalk convenient crossing facilities include functions to reduce vehicular speed and improve vision. The absence of crosswalks can be a movement barrier for pedestrians and potentially contribute to conflicts between pedestrians and motor vehicles. When signals are present, it is vital to allow enough time for all pedestrians to cross the street. Short-signal crosswalk timers can be a movement barrier for pedestrians, and cause hazardous conditions if pedestrians are still crossing when the signals changes. A crosswalk scramble is a crossing treatment which allows pedestrians to cross in all directions, including diagonally across an intersection, while vehicles are stopped in all directions. Traffic calming features are physical features that reduce the negative impact of motor vehicles use by slowing their speed, therefore enhancing walking and bicycling conditions by slowing the speed of traffic and increasing visibility of pedestrians, alerting the driver to potential hazards or providing pedestrian sanctuary.

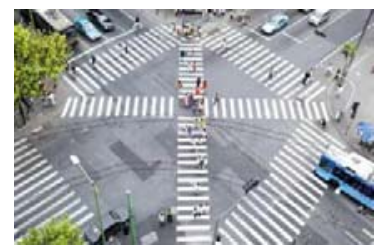

Fig.2
Crosswalk

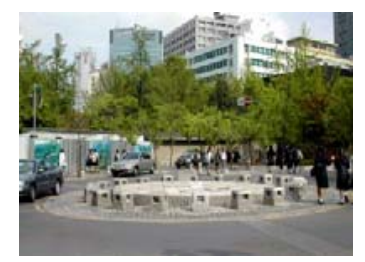

Traffic calming feature

\section{3) Traffic}

The traffic element of the street segment parameter includes prediction of distance to a pedestrian, collision point with a vehicle, degree of pedestrian injury, pedestrian mobility, etc. The vehicle traffic parameter measures factors that are predictive of exposure distance for pedestrian, conflict points, pedestrian injury severity and pedestrian mobility. The number of motor vehicle lanes correlates with traffic volume, posted speed limits, noise and air pollution levels, pedestrian activity, and the levels of social capital reported in a neighborhood. A reduction in the number of lanes can reduce crossing distances, thus reducing exposure of a pedestrian to vehicle interaction.

\section{4) Street Design}

The street design parameter is an important factor for right of way and the local pedestrian environment, and if well constructed and maintained, it enables the safe walking of pedestrians. The width of a sidewalk is a primary factor in determining the level of safety and comfort for pedestrians walking down the streets. A well-maintained sidewalk is crucial to providing a safe pedestrian environment for pedestrians. Sidewalk obstructions can include out-of-place poles or signs, parked cars, trees, and garbage cans. Curbs 
provide a physical separation between motor vehicles and pedestrians. Curbs discourage vehicles from parking on the sidewalk. The more driveway cuts in a street segment, which break up the curb, the greater the potential for vehicles to cause an obstruction to pedestrians and create a potential conflict point with pedestrians. Aspects of the urban landscape contribute to the overall walking experience. Natural elements promote interactions and positive experiences for pedestrians and they can provide a buffer between pedestrian and car traffic. The presence of public seating can encourage leisure walks, especially for the elderly and disabled. Bicycle lanes and parallel parking are two significant streetscape elements that create a buffer for pedestrians.
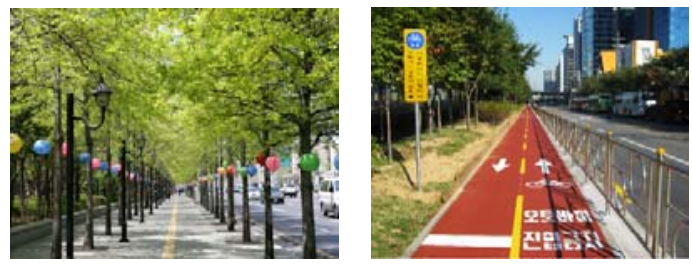

Fig.3 Well-maintained sidewalk Buffer facility

\section{5) Land Use}

The convenient facility and land use parameter includes commercial use and measures the aesthetic aspect of streets. Mixed land use is related to there being a lot of pedestrian traffic and a small amount of vehicle exhaust emissions. Providing a range of artistic and cultural amenities for pedestrians can create a visually interesting environment that attracts pedestrians. Neighborhoods with diverse and mixed land uses can create proximity between residences, employment, and goods and services, reducing vehicle trips and miles traveled and increasing active transportation such as walking and biking. In addition, commercial buildings providing services were found to have a positive effect on pedestrian frequency.

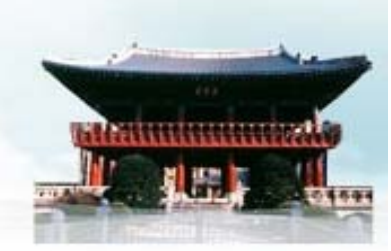

Fig.4

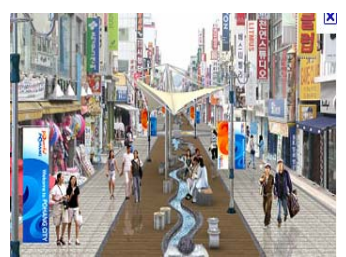

Mixed Land-Use

\section{6) Perceived Safety}

The perceived safety parameter enables strategic design that integrates street lighting and commercial uses to prevent crimes by improving walk-safety awareness through the physical characteristics of the environment. The cleanliness of a street, which includes graffiti, litter garbage and broken glass, as well as pedestrian lighting are important factors found in many walkability audits. Roadway and sidewalk construction can disturb pedestrian flow and create hazards for pedestrians. Construction zones can pose safety issues for pedestrians by blocking the sidewalk with heavy machinery and creating alternate routes that are less accommodating for pedestrians. Abandoned or boarded-up buildings may represent neglect or a lack of adequate funding or impetus to fix neighborhood infrastructure. Abandoned buildings may also increase feelings of pedestrian discomfort.

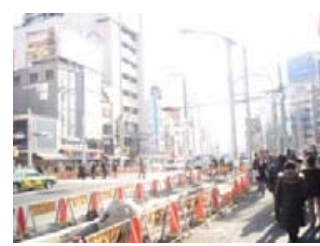

Fig.5

\section{Construction zones}

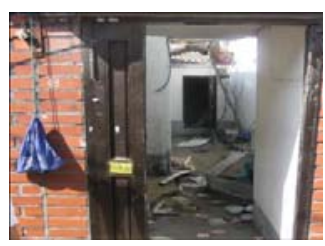

Abandoned buildings

\section{Implementation of Green Score Scheme}

We developed Green Score for street segment parameters and intersection parameters to comprehensively evaluate the service level of pedestrian and vehicular pedestrian environment. First, for pedestrian environment evaluation of intersections, points were assigned to facilities at major crosswalk points interrupted by traffic signals, and vehicle and pedestrian flows, vehicular traffic, and walking and safety facilities were separately measured for the street segment. Second, to evaluate the structural space characteristics of the walking path from the perspective of connectivity and accessibility, space syntax was used and connectivity analysis using Global Integration was conducted. In addition, to measure commercial land use and the aesthetic aspect of streets, relative weights were applied for each type of facility depending on urban 
planning and land use, reflecting a walking preference for mixed land use.

\section{1) Assign Weights with AHP Structure}

As shown in shown Figure 6, we calculated the composite score of the pedestrian environments, Walk Score, and have established AHP structure by calculating weights by individual classes through questionnaire for the specialists. 57 people of civic group and 18 people who specialized in traffic engineering including professor, doctor and master participated in this survey. That is to say, for synthetic evaluation of the pedestrian environment, facility distribution at intersections and on street segments was quantified, along with the connectivity of the walking network. The Analytic Hierarchy Process (AHP) method was used for the survey results to verify the relations between pedestrian satisfaction and evaluation indexes.

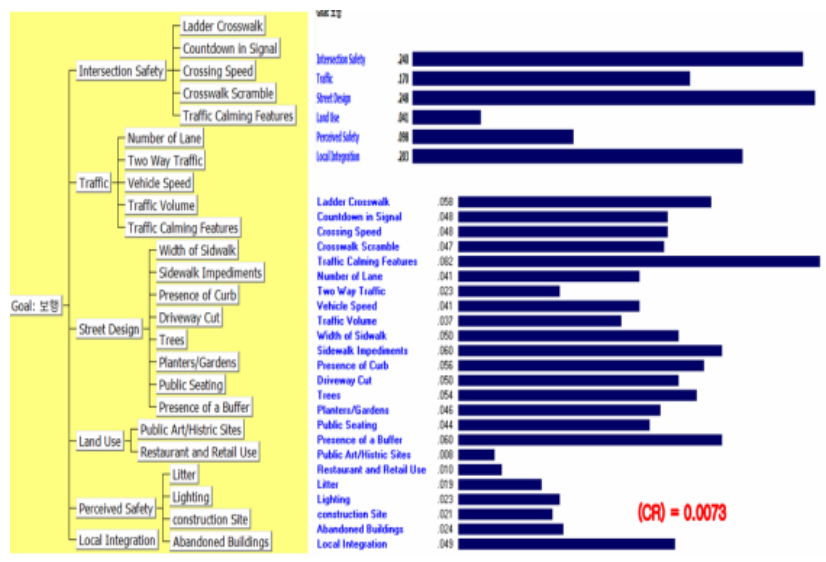

Fig.6 Establish AHP hierarchy and weights

The evaluation results reflect whether each environmental parameter helps in walking. Pedestrian safety is integrated into street segment and intersection design, and the value ranges from 0 to 100 for each parameter

\section{2) Measurement of Walk Accessibility}

We performed an analysis of the geometric accessibility of each subway station using the Space Syntax Model, and the results of the analysis relate to spatial characteristics such as local integration, global integration, and intelligibility. If integration has a high value, fewer movement steps are needed from any space to the relevant space, allowing easy access. The results show that the pedestrian network of the newly developed area has high intelligibility with high recognition and prediction rates in the space. This is because most of the road networks have a mesh structure. Therefore, correlation analysis was conducted in order to identify the relationship between accessibility and road networks. Figure 7 shows the steps of Correlation analysis using Space Syntax Analysis at study area.
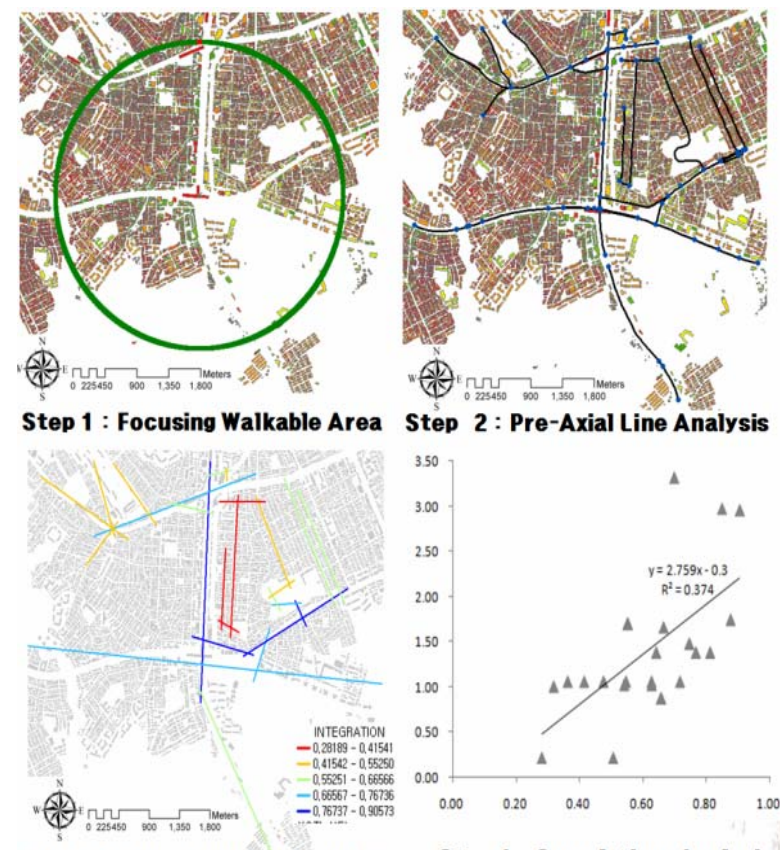

Step 3 : Space Syntax Analysis

Fig.7 Space Syntax Analysis (Intelligibility: 0.6115)

\section{3) Evaluation of the Pedestrian Environment}

Using a street segment and intersection identifier, a visual map was created that incorporated the evaluation results for a selected region consisting of street segment and intersection spaces. The map can be created to indicate the overall pedestrian environment evaluation results, intersection evaluation results, or evaluation results for each sub-parameter. Data for the measurement of evaluation indexes were collected by observations based on the street segment and intersection visualization results. They were then geo-coded, meaning that they were quantified into a score for each link on the traffic map by the Korea Transport Institute. Ultimately, adjusted measurement values were summed and categorized 
according to walking dependency, and these were normalized in the range of 0 to 100 . The resulting scores (synthetic evaluation results) were then mapped for each region as shown in Figure 8.

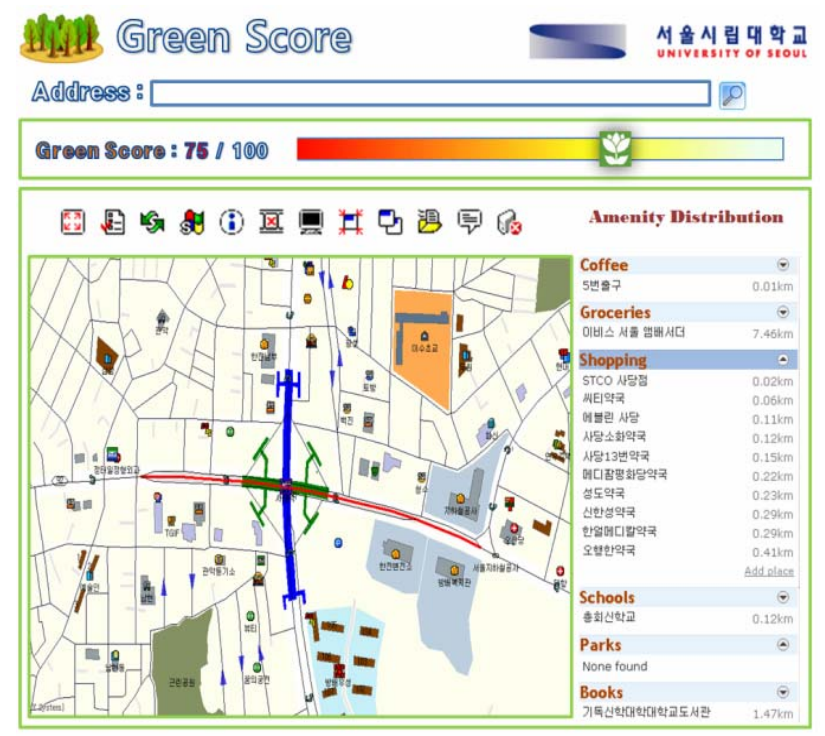

Fig.8 Green Score: Walk Score Evaluation System based on Web GIS Service

\section{Conclusion}

In this study, we developed an evaluation index to measure and quantitatively evaluate pedestrian environmental factors in a city. In order to achieve this goal, we applied the depth-based index used in space syntax theory in assessing the space structure in terms of the connectivity.

For setting the weight values of evaluation criteria obtained from survey, we employed AHP (Analytic Hierarchy Process) to consider relative weights for convenient pedestrian facilities. The computed scores using these criteria are classified and normalized according to the levels of pedestrian dependency and, finally, displayed on a Web-based GIS map. The proposed Green Score is expected to be applicable in the visualization of pedestrian movements and the evaluation of convenience levels of pedestrian environment. Furthermore, this will be used to visualize walking patterns and predict evaluation results of pedestrian environment pleasantness and convenience.

\section{References}

[1] Burnett, A Social History of Housing. David \& Charles , pp. 169-194, 1978.

[2] Hart and Moore, The development of spatial cognition.

In Graduate School of Geography and Department of Psychology, Clark University, pp. 15-86, 1971.

[3] Hillier, B., Space is the Machine, Cambridge University Press, 1996

[4] Hillier, B and Hanson, J, The Social Logic of Space, Cambridge, UK pp. 108-109, 1984.

[5] Jaskiewicz, F., Pedestrian Level of Service Based on Trip Quality. Transportation Research Circular, TRB, 2000. [6] Miller, John S., Biglow, Jeremy A., \& Garber, Nicholas J., Calibrating Pedestrian Level-of-Service Metric with 3-D Visualization, TRR 1705. 2000.

[7] Lee, C. and Moudon, A. V., “The 3Ds + R: Quantifying land use and urban form correlates of walking”, Transportation Research Part D: Transport and Environment, 1, pp. 204 215, 2006.

[8] Lee, Sooil, Lee, Seungjae, Son, Hyeokjun, and Joo, Yongjin. A new approach for the evaluation of Pedestrian Environment,First International Conference on Sustainable Urbanization Proceeding, pp. 1480 1488, 2010.

[9] Moundon, A. V., Lee, C., “An evaluation of environment audit and instruments”, American journal of health promotion, 18, pp. 21 37, 2003.

[10] Penn, A., Hillier, B., Banister, D., and Xu, J.” Configurational modelling of urban movement networks", Environment and Planning B: Planning and Design, 25, 59 -84, 1998.

[11] Saker, Sheila. , Qualitative Evaluation of Comfort Needs in Urban Walkways In Major Activity Centers. TRB Annual Meeting, 2003.

[12] Thambiah, Muraleetharan, “A Study on Evaluation of Pedestrian Level-of-Service along Sidewalks and Crosswalks using Conjoint Analysis”, Journal of Infrastructure Planning, Japan Society of Civil Engineers, 21, 2004.

[13] Thambiah, Muraleetharan. Method to Determine Overall Level-of-Service of Pedestrian on Sidewalks and Crosswalks based on Total Utility Value, TRB Annual Meeting, 2004. 